\title{
The vibration analysis of a nanobeam due to a ramp-type heating under Moore-Gibson-Thompson theory of thermoelasticity
}

\author{
Eman A. N. Al-Lehaibi \\ Department of Mathematics, Al-Lith College, Umm Al-Qura University, Al-Lith, Saudi Arabia \\ E-mail: ealehaibi@uqu.edu.sa
}

Received 30 September 2021; received in revised form 23 December 2021; accepted 30 December 2021 DOI https://doi.org/10.21595/jve.2021.22231

Check for updates

Copyright (C) 2022 Eman A. N. Al-Lehaibi. This is an open access article distributed under the Creative Commons Attribution License, which permits unrestricted use, distribution, and reproduction in any medium, provided the original work is properly cited.

\begin{abstract}
Micro/nanobeams find extensive appreciation due to their employment as resonators. The current study investigates a thermoelastic homogeneous isotropic nanobeam exposed to ramptype heating as a thermal loading. The governing equations of the nanobeam have been deduced in the context of the Moore-Gibson-Thompson thermoelasticity theory. The Laplace transform technique has been applied, while its inversions have been calculated by using Tzou numerical technique with approximation. When a rectangular thermoelastic nanobeam composed of silicon nitride is easily supported, the numerical findings have been confirmed and presented in the figures. It is worth noting that the ramp-time heat parameter has a substantial impact on all of the functions investigated. The parameter that distinguishes the Moore-Gibson-Thompson model from the standard thermoelastic model has a minor influence on temperature increment but has a considerable effect on lateral deflection, strain, and stress distributions.
\end{abstract}

Keywords: thermoelasticity, Moore-Gibson-Thompson, vibration, nanobeam, silicon nitride, ramp-type he.

\section{Introduction}

Nanomechanical resonators have been of great significance. For instance, in communications systems and mechanical electrometers or magnetometers, resonators made of silicone crystal material typically with semi-conductive techniques for the development of $\mathrm{MHz}$ or even $\mathrm{GHz}$ core frequencies may be used for the identification, force, pressure or acceleration sensitive loads [1-3]. Researchers are interested in understanding how the physical and mechanical properties of resonant parameters affect them. Sun et al. [4] test thermoelastic damping (TED) in the microbeam resonators based on generalized thermoelasticity theory with one relaxation time of Lord and Shulman (LS) model [5]. To solve combined thermoelastic equations, he used finite Fourier sinus transformation methods and Laplace methods, which extracted the expressions of fluidity and heat. The deflect vibrates in a simulated state mode when the thermal moment vibrates in the same mode as the deflection at first. In the form of the updated theory of couple tension, Hosseini [6] studied TED and microbeam resonator dynamical behaviour using the theory of thermoelasticity. For the solution of thermoelastic variations and the representation of the beam deflection and thermoelectric moment, the Laplace Transformation technology was adopted. However, the results of the modified pair stress theory on deflection and thermal moment are not evident in their work.

Kumar recently investigated and expanded on the role of the Sun and its impacts on deflection and thermal moment in microbeam resonators, taking into consideration the three-phase of thermoelasticity phase-lags ideas (TPL) [7]. We also address several experiments on thermoelastic beam vibration, as references in the literature indicate [8-13].

Given the foregoing description of beam resonator thermomechanical testing, this study intends to investigate the thermal and dynamic behaviour of simply by using MGT thermoelasticity theory, as recently mentioned by Quintanilla [14]; this is the Moore-Gibson-Thompson (MGT) equation. In this paper, the heat conduction equation is 
described. In reality, this modified heat conduction equation is reached when the relaxation parameter is implemented in the Green-Naghdi type-III model (GN-III) [15, 16]. The coupled thermoelastic problem's governing equations are derived based on the MGT, LS, and GN-III models [16]. Through comparing the findings achieved under the MGT model with those of LS and GN-III models, vibration responses of the deflection and the thermal time are analyzed [16].

In the current work, ramp-type heating as a thermal loading will be applied on thermoelastic isotropic nanobeam. A simplified form of thermoelasticity theory will be used for Moore-Gibson-Thompson. The transform technique for Laplace will be used and its inversions will be computed using the approximation numerical Tzou procedure. The numerical results for a rectangular nanobeam made of silicon nitride will be applied to validate the results.

\section{Basic equations}

A homogeneous thermal isotropic, thermal viscoelastic solid in the Cartesian coordination category Kelvin-Voigt was considered at a uniform temperature $T_{0}$ and initially undeformed. The fundamental differential motion and heat conduction equations for generalized thermoelasticity are based on the thermal control legislation of Non-Fourier.

Without body forces and heat sources, the displacement components $u_{i}$ and absolute temperature $T$ are given by [17].

The equations of motion take the form:

$\sigma_{i j, j}=\rho \ddot{u}_{i}, \quad i, j=1,2,3$.

The constitutive equations with damage mechanics consideration are in the forms [18]:

$\sigma_{i j}=2 \mu e_{i j}+\lambda \delta_{i j} e_{k k}-(3 \lambda+2 \mu) \alpha_{T} \delta_{i j}\left(T-T_{0}\right), \quad i, j=1,2,3$.

The Moore-Gibson-Thompson (MGT) heat conduction equation takes the form $[14,18,19]$ :

$$
\left(K \dot{T}_{, i i}+K^{*} T_{, i i}\right)=\left(1+\tau_{q} \frac{\partial}{\partial t}\right)\left[\rho C_{v} \ddot{T}+T_{o}(3 \lambda+2 \mu) \alpha_{T} \delta_{i j} \ddot{e}_{i j}\right], \quad i, j=1,2,3 .
$$

The MGT theory is the widespread thermoelasticity of the Lord and Shulman theory (LS) and Type-III (GN-III) thermoelasticity of Green-Naghdi [16]. The thermoelastic Eq. (3) therefore gives:

- If $K^{*}=0$ and $\tau_{q} \neq 0$, we obtain the Lord-Shulman (LS) model [19].

- If $K^{*} \neq 0$ and $\tau_{q}=0$, we obtain the Green-Naghdi (GN-III) model [16, 19].

- If $K^{*} \neq 0$ and $\tau_{q} \neq 0$, we obtain the Moore-Gibson-Thompson (MGT) model [14].

The deformation-displacement relations are in the form:

$e_{i j}=\frac{1}{2}\left(u_{i, j}+u_{j, i}\right), \quad i, j=1,2,3$,

where $i, j=x, y, z, \beta=(3 \lambda+2 \mu) \alpha_{T}, \alpha_{T}$ is the coefficient of linear thermal expansion, $\rho$ is the density, $\tau_{q}$ is the thermal relaxation time, $\lambda, \mu$ Lamè's parameter in the usual case, $\delta_{i j}$ is the Kronecker delta symbol, $K$ is the thermal conductivity, $K^{*}$ is the conductivity rate parameter, and $C_{v}$ is the specific heat at constant strain, and for stable solution $K>K^{*} \tau_{q}[14,19]$.

\section{Problem formulation}

We consider a thin thermoelastic nanobeam of length $\ell \quad(0 \leq x \leq \ell)$, width $b$ $(-b / 2 \leq y \leq b / 2)$, and thickness $h(-h / 2 \leq z \leq h / 2)$ with small flexural deflections. The 
three axes $x, y$, and $z$ are defined along the longitudinal, width, and thickness directions of the beam, respectively.

In the state of equilibrium, the nanobeam is unstrained, unstressed, and without any mechanical damping. Moreover, the reference temperature is $T_{0}$ everywhere [6].

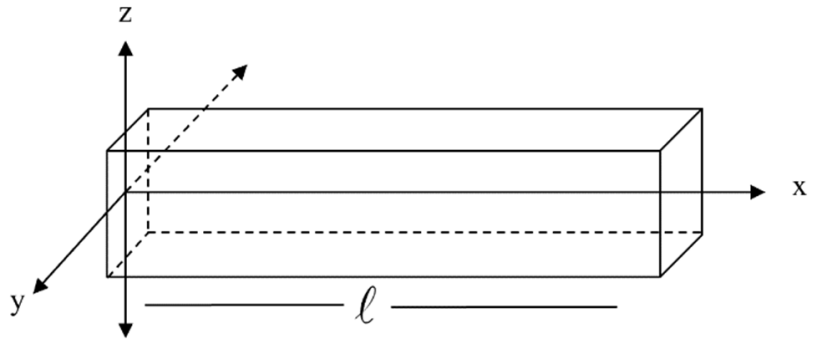

Fig. 1. Rectangular thermoelastic nanobeam

In this work, the well-known Euler-Bernoulli equation [20] is adopted, therefore any plane cross-section that is at first perpendicular to the axis of the beam stays flat and perpendicular during bending to the neutral surface as in Fig. 1.

Hence, the displacement components take the forms:

$u(x, z, t)=\left(-z \frac{\partial w(x, t)}{\partial x}, 0, w(x, t)\right)$

where $w(x, t)$ is defined as the lateral deflection.

The flexural moment of the nanobeam cross-section is given by:

$M(x, t)=I(\lambda+2 \mu) \frac{\partial^{2} w(x, t)}{\partial x^{2}}+(3 \lambda+2 \mu) \alpha_{T} M_{T}(x, t)$,

where $I=b h^{3} / 12$ gives the moment of inertia of the nanobeam cross-section around the $x$-axis and $M_{T}$ defines the thermal moment of the nanobeam about the $x$-axis, and it is given by:

$M_{T}(x, t)=\int_{-h / 2}^{h / 2} b T(x, z, t) z d z$

As a result, the differential equation of thermally induced lateral vibration of the beam may be written as [20]:

$\frac{\partial^{4} w(x, t)}{\partial x^{4}}+\frac{\rho A}{(\lambda+2 \mu) I} \frac{\partial^{2} w(x, t)}{\partial t^{2}}+\frac{(3 \lambda+2 \mu) \alpha_{T}}{(\lambda+2 \mu) I} \frac{\partial^{2} M_{T}(x, t)}{\partial x^{2}}=0$,

where $A=h b$ is the cross-section area.

The non-Fourier heat conduction Eq. (3) takes the form [20]:

$\left(\frac{K^{*}}{K}+\frac{\partial}{\partial t}\right)\left(\frac{\partial^{2} T(x, z, t)}{\partial x^{2}}+\frac{\partial^{2} T(x, z, t)}{\partial z^{2}}\right)=\left(\frac{\partial^{2}}{\partial t^{2}}+\tau_{q} \frac{\partial^{3}}{\partial t^{3}}\right)\left[\frac{\rho C_{v}}{K} T+\frac{T_{o}(3 \lambda+2 \mu) \alpha_{T}}{K} e\right]$,
$\sigma(x, z, t)=(\lambda+2 \mu) e(x, z, t)-(3 \lambda+2 \mu) \alpha_{T}\left(T(x, z, t)-T_{0}\right)$,

where: 
$e(x, z, t)=e_{x x}(x, z, t)+e_{y y}(x, z, t)+e_{z z}(x, z, t)$,

is the volumetric strain which gives from Eq. (4) and Eq. (5) that:

$e(x, z, t)=-z \frac{\partial^{2} w(x, t)}{\partial x^{2}}$

The nanobeam has no heat flow across the upper and lower surfaces of the beam, thus, $\left.\frac{\partial T(x, z, t)}{\partial z}\right|_{z=\frac{ \pm h}{2}}=0$.

For a very thin nanobeam, we assume the temperature varies in terms of a " $\sin (p z)$ " function through the thickness direction as follows [21]:

$\theta(x, z, t)=T(x, z, t)-T_{0}=\sin (p z) \vartheta(x, t)$,

where $\theta(x, z, t)$ is the temperature increment and $p=\pi / h$.

Hence, form Eq. (7), Eq. (8), and Eq. (13), we obtain:

$\frac{\partial^{4} w(x, t)}{\partial x^{4}}+\frac{12 \rho}{(\lambda+2 \mu) h^{2}} \frac{\partial^{2} w(x, t)}{\partial t^{2}}+\frac{12(3 \lambda+2 \mu) \alpha_{T}}{(\lambda+2 \mu) h^{3}} \frac{\partial^{2} \vartheta(x, t)}{\partial x^{2}} \int_{-h / 2}^{h / 2} \sin (p z) z d z=0$.

From Eq. (9) and Eq. (13), we get:

$$
\begin{aligned}
& \left(\delta+\frac{\partial}{\partial t}\right)\left(\frac{\partial^{2} \vartheta(x, t)}{\partial x^{2}}-p^{2} \vartheta(x, t)\right) \sin (p z) \\
& \quad=\left(\frac{\partial^{2}}{\partial t^{2}}+\tau_{q} \frac{\partial^{3}}{\partial t^{3}}\right)\left(\varepsilon \vartheta(x, t) \sin (p z)-\frac{T_{o}(3 \lambda+2 \mu) \alpha_{T}}{K} \frac{\partial^{2} w(x, t)}{\partial x^{2}} z\right), \\
& \sigma(x, z, t)=(\lambda+2 \mu) e(x, z, t)-(3 \lambda+2 \mu) \alpha_{T} \theta(x, z, t),
\end{aligned}
$$

where $\varepsilon=\rho C_{v} / K$ and $\delta=K^{*} / K$.

Thus, we have:

- When $\delta=0, \tau_{q} \neq 0$, we obtain the Lord-Shulman (LS) model.

- When $\delta \neq 0, \tau_{q}=0$, we receive the Green-Naghdi type-III (GN-III) model.

- When $\delta \neq 0, \tau_{q} \neq 0$, we get the Moore-Gibson-Thompson (MGT) model.

Hence, equations and Eq. (14) gives:

$\frac{\partial^{4} w(x, t)}{\partial x^{4}}+\frac{12 \rho}{(\lambda+2 \mu) h^{2}} \ddot{w}(x, t)+\frac{12(3 \lambda+2 \mu) \alpha_{T}}{(\lambda+2 \mu) h^{3}} \frac{\partial^{2} \vartheta(x, t)}{\partial x^{2}} \int_{-h / 2}^{h / 2} z \sin (p z) d z=0$.

After executing the integrations, Eq. (17) takes the form:

$\frac{\partial^{4} w(x, t)}{\partial x^{4}}+\frac{12 \rho}{(\lambda+2 \mu) h^{2}} \ddot{w}(x, t)+\frac{24(3 \lambda+2 \mu) \alpha_{T}}{(\lambda+2 \mu) h \pi^{2}} \frac{\partial^{2} \vartheta(x, t)}{\partial x^{2}}=0$.

In Eq. (15), we multiply both sides by $z$ and integrate for $z$ from $(-h / 2)$ to $(h / 2)$, then we obtain: 


$$
\begin{aligned}
(\delta & \left.+\frac{\partial}{\partial t}\right)\left[\frac{\partial^{2} \vartheta(x, t)}{\partial x^{2}}-p^{2} \vartheta(x, t)\right] \\
& =\left(\frac{\partial^{2}}{\partial t^{2}}+\tau_{q} \frac{\partial^{3}}{\partial t^{3}}\right)\left(\varepsilon \vartheta(x, t)-\frac{T_{o}(3 \lambda+2 \mu) \alpha_{T} h \pi^{2}}{24 K} \frac{\partial^{2} w(x, t)}{\partial x^{2}}\right)
\end{aligned}
$$

For simplicity, the following non-dimensional variables will be applied [19], [22]:

$$
\begin{aligned}
& \left(t^{\prime}, \tau_{q}^{\prime}\right)=\frac{\varepsilon c_{0}^{3}}{\ell}\left(t, \tau_{q}\right), \quad\left(x^{\prime}, w^{\prime}, h^{\prime}\right)=\frac{\varepsilon c_{0}^{2}}{\ell}(x, w, h), \\
& \sigma^{\prime}=\frac{\sigma}{(\lambda+2 \mu)}, \quad \vartheta^{\prime}=\frac{\vartheta}{T_{0}}, \quad \delta^{\prime}=\frac{\ell}{\varepsilon c_{0}^{3}} \delta
\end{aligned}
$$

where $c_{0}^{2}=\lambda+2 \mu / \rho$.

Then, we have the following equations:

$\left(\delta+\frac{\partial}{\partial t}\right)\left[\frac{\partial^{2} \vartheta(x, t)}{\partial x^{2}}-p^{2} \vartheta(x, t)\right]=\left(\frac{\partial^{2}}{\partial t^{2}}+\tau_{q} \frac{\partial^{3}}{\partial t^{3}}\right)\left(\varepsilon_{1} \vartheta(x, t)-\varepsilon_{2} \frac{\partial^{2} w(x, t)}{\partial x^{2}}\right)$,

$\frac{\partial^{4} w(x, t)}{\partial x^{4}}+\varepsilon_{3} \ddot{w}(x, t)+\varepsilon_{4} \frac{\partial^{2} \vartheta(x, t)}{\partial x^{2}}=0$,

$\sigma(x, z, t)=e(x, z, t)-\varepsilon_{5} \theta(x, z, t)$,

where $\varepsilon_{1}=\varepsilon \ell / c_{0}, \quad \varepsilon_{2}=(3 \lambda+2 \mu) \alpha_{T} \ell h \pi^{2} / 24 c_{0} K, \quad \varepsilon_{3}=12 / h^{2}, \quad \varepsilon_{4}=24 \varepsilon_{5} / h \pi^{2}$, and $\varepsilon_{5}=(3 \lambda+2 \mu) \alpha_{T} T_{0} /(\lambda+2 \mu)$.

(The primes have been cancelled for convenience).

\section{Formulation of the problem by using Laplace transform}

The Laplace transform will be applied for Eq. (21) and Eq. (22), which is defined by the following formula:

$\bar{f}(s)=\int_{0}^{\infty} f(t) e^{-s t} d t$.

The Laplace transform inversions could be obtained by using the following formula:

$L^{-1}(\bar{f}(s))=f(t) \approx \frac{e^{\kappa t}}{t}\left[\frac{1}{2} \bar{f}(\kappa)+\operatorname{Re} \sum_{n=1}^{N}(-1)^{n} \bar{f}\left(\kappa+\frac{i n \pi}{t}\right)\right]$,

where $i$ is an imaginary number unit and Re is the real part.

For faster convergence, many numerical experiments have concluded that the value $\kappa$ satisfies the relation $\kappa t \approx 4.7$ Tzou $[22,23]$.

Hence:

$\frac{d^{2} \bar{\vartheta}}{d x^{2}}-\alpha_{2} \bar{\vartheta}=-\alpha_{1} \varepsilon_{2} \frac{d^{2} \bar{w}}{d x^{2}}$

$\frac{d^{4} \bar{w}}{d x^{4}}+\varepsilon_{3} s^{2} \bar{w}+\varepsilon_{4} \frac{d^{2} \bar{\vartheta}}{d x^{2}}=0$,

$\bar{\sigma}=\bar{e}-\varepsilon_{5} \bar{\theta}$, 
$\bar{e}=-z \frac{d^{2} \bar{w}}{d x^{2}}$

where $\alpha_{1}=\left(s^{2}+\tau_{q} s^{3}\right) /(\delta+s)$ and $\alpha_{2}=\left(p^{2}+\alpha_{1} \varepsilon_{1}\right)$.

We can re-write Eq. (26) and Eq. (27) to be in the forms:

$\left(D^{2}-\alpha_{2}\right) \bar{\vartheta}+\alpha_{1} \varepsilon_{2} D^{2} \bar{w}=0$,

$\varepsilon_{4} D^{2} \bar{\vartheta}+\left(D^{4}+\varepsilon_{3} s^{2}\right) \bar{w}=0$,

where $D^{r}=d^{r} / d x^{r}$.

After elimination, we get:

$\left[D^{6}-L D^{4}+M D^{2}-N\right]\{\bar{\vartheta}, \bar{w}\}=0$,

where $L=\left(\alpha_{2}+\alpha_{1} \varepsilon_{2} \varepsilon_{4}\right), M=\varepsilon_{3} s^{2}$, and $N=\varepsilon_{3} \alpha_{2} s^{2}$.

The general solutions of Eq. (30) and Eq. (31) will take the forms:

$\bar{\vartheta}(x, s)=\sum_{i=1}^{3}\left(A_{i} e^{k_{i} x}+B_{i} e^{-k_{i} x}\right)$,

$\bar{w}(x, s)=\sum_{i=1}^{3} C_{i}\left(A_{i} e^{k_{i} x}+B_{i} e^{-k_{i} x}\right)$,

where $A_{i}, B_{i}$, and $C_{i}$ are unknown parameters to be determined, and the roots of the characteristic equation are $\pm k_{1}, \pm k_{2}$, and $\pm k_{3}$ which satisfy the following characteristic equation:

$k^{6}-L k^{4}+M k^{2}-N=0$.

To get the parameters, $C_{i}$ we will substitute from Eq. (33) and Eq. (34) into the Eq. (30). Hence, we have:

$C_{i}=\frac{\alpha_{2}-k_{i}^{2}}{\alpha_{1} \varepsilon_{2} k_{i}^{2}}$

which gives:

$\bar{w}(x, s)=\frac{1}{\alpha_{1} \varepsilon_{2}} \sum_{i=1}^{3} \frac{\left(\alpha_{2}-k_{i}^{2}\right)}{k_{i}^{2}}\left(A_{i} e^{k_{i} x}+B_{i} e^{-k_{i} x}\right)$.

To calculate the parameters $A_{i}, i=1,2,3$, we must use the boundary conditions.

We consider that the first end of the beam $x=0$ is thermally loaded by a function $g(t)$ and simply supported as follows:

$w(0, t)=\frac{\partial^{2} w(0, t)}{\partial x^{2}}=0, \quad \vartheta(0, t)=\vartheta_{0} g(t)$,

where $\vartheta_{0}$ is constant.

The second end of the beam $x=\ell$ is simply supported, and zero temperature increment:

$w(\ell, t)=\frac{\partial^{2} w(\ell, t)}{\partial x^{2}}=\vartheta(\ell, t)=0$. 
Applying the Laplace transform, we get:

$\bar{w}(0, s)=\frac{\partial^{2} \bar{w}(0, s)}{\partial x^{2}}=0$,

$\bar{\vartheta}(0, s)=\vartheta_{0} \bar{g}(s)$

$\bar{w}(\ell, s)=\frac{\partial^{2} \bar{w}(\ell, s)}{\partial x^{2}}=\bar{\vartheta}(\ell, s)=0$.

Then we obtain the system of linear equations shown below:

$\sum_{i=1}^{3}\left(A_{i}+B_{i}\right)=\vartheta_{0} \bar{g}(s)$,
$\sum_{i=1}^{3} \frac{\left(\alpha_{2}-k_{i}^{2}\right)}{k_{i}^{2}}\left(A_{i}+B_{i}\right)=0$,
$\sum_{i=1}^{3}\left(\alpha_{2}-k_{i}^{2}\right)\left(A_{i}+B_{i}\right)=0$,
$\sum_{i=1}^{3}\left(A_{i} e^{k_{i} \ell}+B_{i} e^{-k_{i} \ell}\right)=0$,
$\sum_{i=1}^{3} \frac{\left(\alpha_{2}-k_{i}^{2}\right)}{k_{i}^{2}}\left(A_{i} e^{k_{i} \ell}+B_{i} e^{-k_{i} \ell}\right)=0$,
$\sum_{i=1}^{3}\left(\alpha_{2}-k_{i}^{2}\right)\left(A_{i} e^{k_{i} \ell}+B_{i} e^{-k_{i} \ell}\right)=0$.

A mathematical software MAPLE 2020 has been used to solve the above system and obtain the constants $A_{1}, A_{2}, A_{3}$.

Hence, we get:

$$
\begin{gathered}
\bar{\theta}(x, z, s)=\frac{\vartheta_{0} \bar{g}(s) \sin (p z)}{\alpha_{2}}\left[\frac{\left(k_{2}^{2}-\alpha_{2}\right)\left(k_{3}^{2}-\alpha_{2}\right) k_{1}^{2}}{\left(k_{1}^{2}-k_{2}^{2}\right)\left(k_{1}^{2}-k_{3}^{2}\right) \sinh \left(k_{1} \ell\right)} \sinh \left(k_{1}(\ell-x)\right)\right. \\
+\frac{\left(k_{1}^{2}-\alpha_{2}\right)\left(k_{3}^{2}-\alpha_{2}\right) k_{2}^{2}}{\left(k_{2}^{2}-k_{1}^{2}\right)\left(k_{2}^{2}-k_{3}^{2}\right) \sinh \left(k_{2} \ell\right)} \sinh \left(k_{2}(\ell-x)\right) \\
\left.+\frac{\left(k_{2}^{2}-\alpha_{2}\right)\left(k_{1}^{2}-\alpha_{2}\right) k_{3}^{2}}{\left(k_{3}^{2}-k_{1}^{2}\right)\left(k_{3}^{2}-k_{2}^{2}\right) \sinh \left(k_{3} \ell\right)} \sinh \left(k_{3}(\ell-x)\right)\right]
\end{gathered}
$$

The lateral deflection is:

$$
\begin{aligned}
& \bar{w}(x, s)=-\vartheta_{0} \bar{g}(s) \alpha^{2} \varepsilon_{4}\left[\frac{\sinh \left(k_{1}(\ell-x)\right)}{\left(k_{1}^{2}-k_{2}^{2}\right)\left(k_{1}^{2}-k_{3}^{2}\right) \sinh \left(k_{1} \ell\right)}\right. \\
& \left.\quad+\frac{\sinh \left(k_{2}(\ell-x)\right)}{\left(k_{2}^{2}-k_{1}^{2}\right)\left(k_{2}^{2}-k_{3}^{2}\right) \sinh \left(k_{2} \ell\right)}+\frac{\sinh \left(k_{3}(\ell-x)\right)}{\left(k_{3}^{2}-k_{1}^{2}\right)\left(k_{3}^{2}-k_{2}^{2}\right) \sinh \left(k_{3} \ell\right)}\right],
\end{aligned}
$$

and the deformation from Eq. (29) and Eq. (50) takes the form: 


$$
\begin{aligned}
& \bar{e}(x, z, s)=z \vartheta_{0} \bar{g}(s) \alpha^{2} \varepsilon_{4}\left[\frac{k_{1}^{2} \sinh \left(k_{1}(\ell-x)\right)}{\left(k_{1}^{2}-k_{2}^{2}\right)\left(k_{1}^{2}-k_{3}^{2}\right) \sinh \left(k_{1} \ell\right)}\right. \\
& \left.+\frac{k_{2}^{2} \sinh \left(k_{2}(\ell-x)\right)}{\left(k_{2}^{2}-k_{1}^{2}\right)\left(k_{2}^{2}-k_{3}^{2}\right) \sinh \left(k_{2} \ell\right)}+\frac{k_{3}^{2} \sinh \left(k_{3}(\ell-x)\right)}{\left(k_{3}^{2}-k_{1}^{2}\right)\left(k_{3}^{2}-k_{2}^{2}\right) \sinh \left(k_{3} \ell\right)}\right]
\end{aligned}
$$

which complete the solution in the Laplace transform domain.

\section{Numerical Results}

For the thermal loading, we considered the ramp-type heating, which takes the form [22]:

$$
g(t)= \begin{cases}\frac{t}{t_{0}}, & 0 \leq t<t_{0} \\ 1, & t \geq t_{0}\end{cases}
$$

By applying Laplace transform on the above equation, we get:

$$
\bar{g}(s)=\frac{1-e^{-t_{0} s}}{t_{0} s^{2}} .
$$

Finally, Eq. (49), Eq. (50), Eq. (51), and Eq. (53) represent the solutions in the Laplace transform domain. For the numerical results purpose, the silicon nitride has been taken as the thermoelastic material for which we considered the following values of the different physical constants [17, 20, 24]: $k=43.5 \mathrm{~W} /(\mathrm{m} \mathrm{K}), \alpha_{T}=2.7110^{-6} \mathrm{~K}^{-1}, \rho=3200 \mathrm{~kg} / \mathrm{m}^{3}, T_{0}=293 \mathrm{~K}$, $C_{v}=630 \mathrm{~J} /(\mathrm{kg} \mathrm{K}), \lambda=217 \times 10^{9} \mathrm{~N} / \mathrm{m}^{2}, \mu=108 \times 10^{9} \mathrm{~N} / \mathrm{m}^{2}, \tau_{0}=4.32 \times 10^{-13} \mathrm{~s}$.

The aspect ratios of the nanobeam have been fixed as $\ell / h=8$ and $b=h / 2$. For the nanobeam, we will assume the range of the nanobeam length is $\ell(1-100) \times 10^{-12} \mathrm{~m}$, and the original time $t$ and the relaxation time $\tau_{q}$ are of order $10^{-12} \mathrm{sec}$ and $10^{-14} \mathrm{sec}$, respectively.

The figures will be prepared by using the non-dimensional variables for nanobeam as $\ell=0.5$, $\theta_{0}=1.0, z=h / 4$, and $t=1.0$.

\section{Discussion}

Figs. 2-5 represent the temperature increment, lateral deflection, strain, and stress distributions for the three studied models: Lord-Shulman (LS), Green-Naghdi type-III (GN-III), and the newest one Moore-Gibson-Thompson (MGT) when $t=t_{0}$.

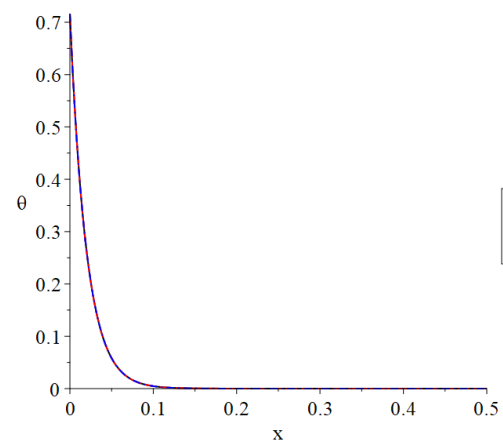

Fig. 2. The temperature increment for different models when $t=t_{0}$

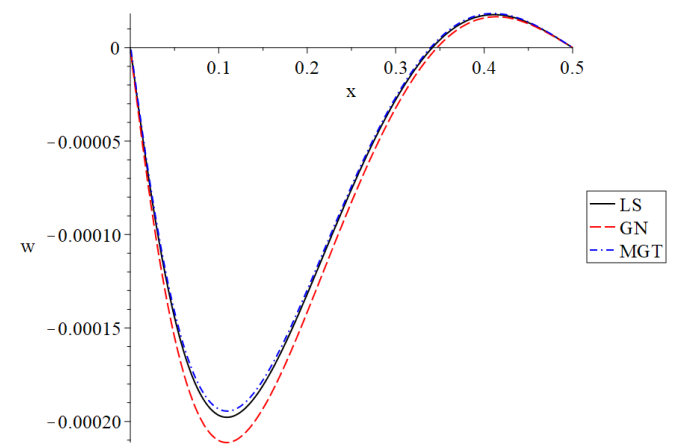

Fig. 3. The lateral deflection for different models when $t=t_{0}$ 
Fig. 2 shows that the three models offer the same behaviour and values for the temperature increment, where the three curves are very close from the beginning up to the end.

Fig. 3 shows that the parameters $\delta$ and $\tau_{q}$ have significant effects on the lateral deflection distributions. The absolute values of the lateral deflection take the following order:

$|w(G N)|>|w(L S)|>|w(M G T)|$.

Fig. 4 shows that the parameters $\delta$ and $\tau_{q}$ have significant effects on the strain distributions. The absolute values of the strain take the following order:

$|e(G N)|>|e(L S)|>|e(M G T)|$.

Fig. 5 shows that the parameters $\delta$ and $\tau_{q}$ have significant effects on the stress distributions. The absolute values of the strain take the following order:

$|\sigma(G N)|>|\sigma(L S)|>|\sigma(M G T)|$.

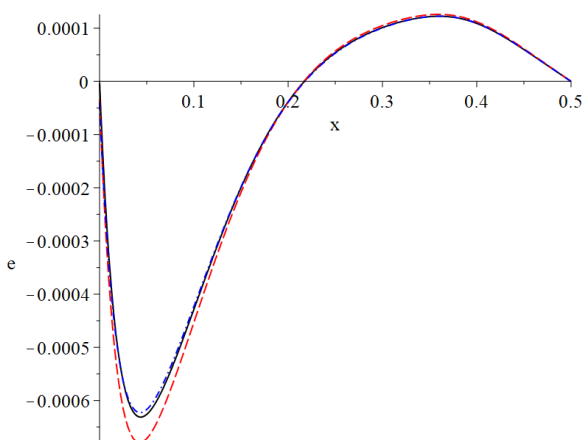

Fig. 4. The strain for different models when $t=t_{0}$

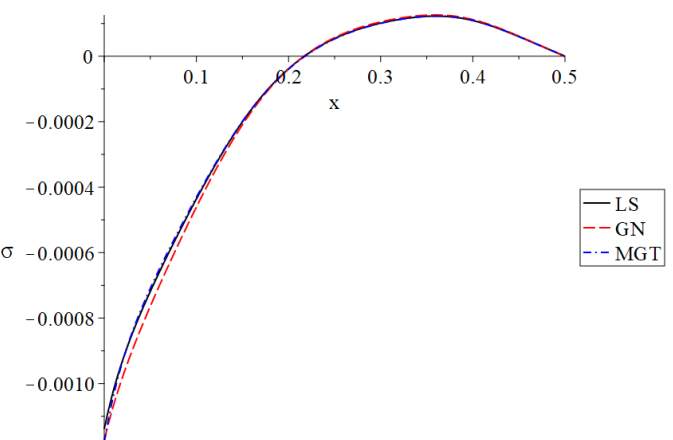

Fig. 5. The stress for different models when $t=t_{0}$

Figs. 6-9 show the temperature increment, lateral deflection, strain, and stress distributions for the three studied models: Lord-Shulman (LS), Green-Naghdi type-III (GN-III), and the newest one Moore-Gibson-Thompson (MGT) when $t<t_{0}$. Those figures show the same behaviour of the distributions in Figs. 2-5 for the temperature increment, lateral deflection, strain, and stress but with different values due to the ramp-time heat parameter $t_{0}$.

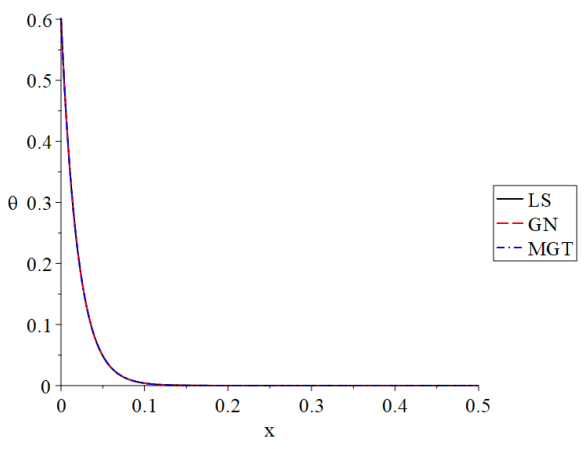

Fig. 6. The temperature increment for different models when $t<t_{0}$

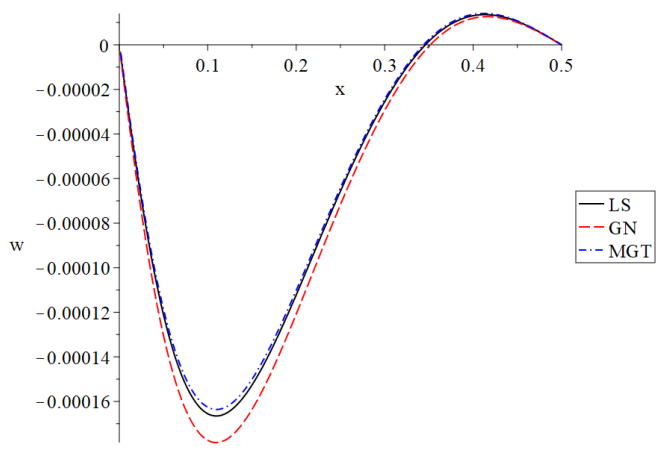

Fig. 7. The lateral deflection for different models when $t<t_{0}$

We noted that an increase in the value of the ramp-time heat parameter leads to a decrease in the temperature increment, lateral deflection, strain, and stress of the nanobeam. 
Thus, the ramp-time heat parameter $t_{0}$ has significant effects on all the studied functions and the thermomechanical waves' propagation through the nanobeam.

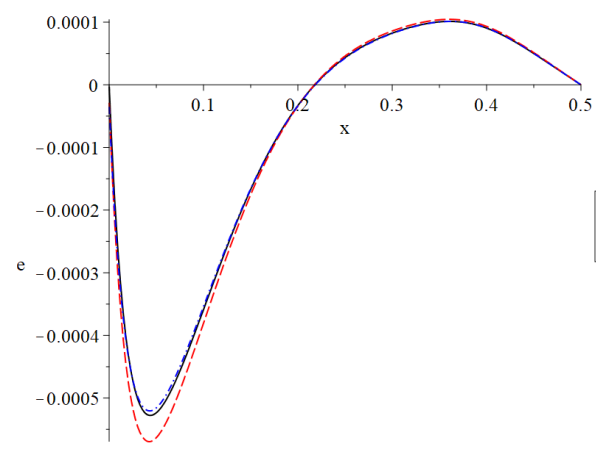

Fig. 8. The strain for different models when $t<t_{0}$

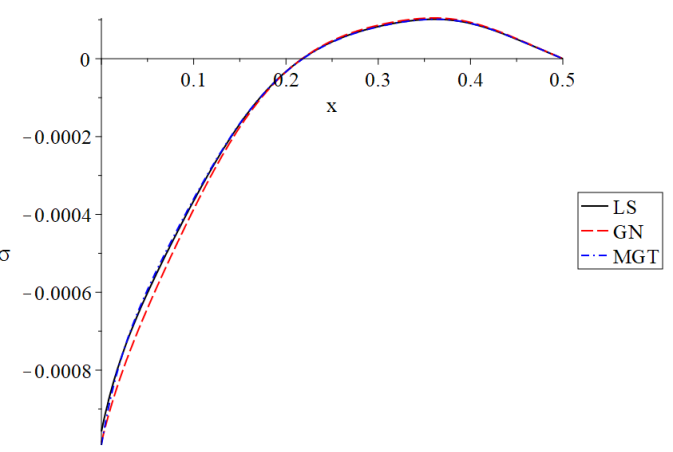

Fig. 9. The stress for different models when $t<t_{0}$

\section{Conclusions}

The current study looks at the temperature increment, lateral deflection, strain, and stress of a simply supported nanobeam resonator heated using a ramp. In terms of dimensionless temperature increment, lateral deflection, strain, and stress functions, the governing equations for three distinct coupled thermoelasticity theories (LS, GN-III, and MGT) of nanobeams have been developed. The following are the major achievements of this work:

1) The temperature increment under the three studied models is approximately the same.

2) The absolute values of the vibration, strain, and stress in the context of the GN model are greater than its values under the LS model, and they have smaller values under the MGT model.

3) As the uniform load on the upper surface of the nanobeam rises, so do the deflection amplitudes and thermal momentum.

4) As the ramp-time heat parameter is increased, the temperature rise, lateral deflection, strain, and stress decrease.

5) The relaxation parameter has a considerable effect on the vibration of the lateral deflection, strain, and stress distributions.

6) In future work, this work could be extended to include other types of thermal and mechanical loading and different types of nanobeams such as circular nanobeams.

\section{References}

[1] H. Kumar and S. Mukhopadhyay, "Thermoelastic damping analysis in microbeam resonators based on Moore-Gibson-Thompson generalized thermoelasticity theory," Acta Mechanica, Vol. 231, No. 7, pp. 3003-3015, Jul. 2020, https://doi.org/10.1007/s00707-020-02688-6

[2] C. Zhang, G. Xu, and Q. Jiang, "Analysis of the air-damping effect on a micromachined beam resonator," Mathematics and Mechanics of Solids, Vol. 8, No. 3, pp. 315-325, Jun. 2003, https://doi.org/10.1177/1081286503008003006

[3] A. E. Green and K. A. Lindsay, "Thermoelasticity," Journal of Elasticity, Vol. 2, No. 1, pp. 1-7, 1972.

[4] Y. Sun, D. Fang, and A. K. Soh, "Thermoelastic damping in micro-beam resonators," International Journal of Solids and Structures, Vol. 43, No. 10, pp. 3213-3229, May 2006, https://doi.org/10.1016/j.ijsolstr.2005.08.011

[5] H. W. Lord and Y. Shulman, "A generalized dynamical theory of thermoelasticity," Journal of the Mechanics and Physics of Solids, Vol. 15, No. 5, pp. 299-309, Sep. 1967, https://doi.org/10.1016/0022-5096(67)90024-5

[6] E. Kazemnia Kakhki, S. M. Hosseini, and M. Tahani, "An analytical solution for thermoelastic damping in a micro-beam based on generalized theory of thermoelasticity and modified couple stress theory," Applied Mathematical Modelling, Vol. 40, No. 4, pp. 3164-3174, Feb. 2016, https://doi.org/10.1016/j.apm.2015.10.019 
[7] R. Kumar and R. Kumar, "Effects of phase lags on thermoelastic damping in micro-beam resonators," International Journal of Structural Stability and Dynamics, Vol. 19, No. 9, p. 1971005, Sep. 2019, https://doi.org/10.1142/s0219455419710056

[8] H. M. Youssef and K. A. Elsibai, "State-space approach to vibration of gold nano-beam induced by ramp type heating," Nano-Micro Letters, Vol. 2, No. 3, pp. 139-147, Sep. 2010, https://doi.org/10.1007/bf03353632

[9] H. M. Youssef, A. A. El-Bary, and K. A. Elsibai, "Vibration of gold nano beam in context of twotemperature generalized thermoelasticity subjected to laser pulse," Latin American Journal of Solids and Structures, Vol. 11, No. 13, pp. 2460-2482, 2014.

[10] H. M. Youssef and N. A. Alghamdi, "Thermoelastic damping in nanomechanical resonators based on two-temperature generalized thermoelasticity theory," Journal of Thermal Stresses, Vol. 38, No. 12 , pp. 1345-1359, Dec. 2015, https://doi.org/10.1080/01495739.2015.1073541

[11] E. N. Al-Lehaibi and H. Youssef, "State-space approach to nano-beam with variable material properties," Advanced Science, Engineering and Medicine, Vol. 8, No. 5, pp. 412-420, Jun. 2016, https://doi.org/10.1166/asem.2016.1865

[12] H. M. Youssef, K. A. Elsibai, and A. A. El-Bary, "Effect of the speed, the rotation and the magnetic field on the Q-factor of an axially clamped gold micro-beam," Meccanica, Vol. 52, No. 7, pp. 1685-1694, May 2017, https://doi.org/10.1007/s11012-016-0498-8

[13] H. M. Youssef and A. A. El-Bary, "The reference temperature dependence of Young's modulus of twotemperature thermoelastic damping of gold nano-beam," Mechanics of Time-Dependent Materials, Vol. 22, No. 4, pp. 435-445, Nov. 2018, https://doi.org/10.1007/s11043-017-9365-9

[14] R. Quintanilla, "Moore-Gibson-Thompson thermoelasticity," Mathematics and Mechanics of Solids, Vol. 24, No. 12, pp. 4020-4031, Dec. 2019, https://doi.org/10.1177/1081286519862007

[15] A. E. Green and P. M. Naghdi, "A re-examination of the basic postulates of thermomechanics," Proceedings of the Royal Society of London. Series A: Mathematical and Physical Sciences, Vol. 432, No. 1885, pp. 171-194, Feb. 1991, https://doi.org/10.1098/rspa.1991.0012

[16] A. E. Green and P. M. Naghdi, "Thermoelasticity without energy dissipation," Journal of Elasticity, Vol. 31, No. 3, pp. 189-208, Jun. 1993, https://doi.org/10.1007/bf00044969

[17] D. Grover, "Transverse vibrations in micro-scale viscothermoelastic beam resonators," Archive of Applied Mechanics, Vol. 83, No. 2, pp. 303-314, Feb. 2013, https://doi.org/10.1007/s00419-012-0656-y

[18] K. Saanouni, J. F. Mariage, A. Cherouat, and P. Lestriez, "Numerical prediction of discontinuous central bursting in axisymmetric forward extrusion by continuum damage mechanics," Computers and Structures, Vol. 82, No. 27, pp. 2309-2332, Oct. 2004, https://doi.org/10.1016/j.compstruc.2004.05.018

[19] R. Quintanilla, "Moore-Gibson-Thompson thermoelasticity with two temperatures," Applications in Engineering Science, Vol. 1, p. 100006, Mar. 2020, https://doi.org/10.1016/j.apples.2020.100006

[20] D. Grover, "Viscothermoelastic vibrations in micro-scale beam resonators with linearly varying thickness," Canadian Journal of Physics, Vol. 90, No. 5, pp. 487-496, May 2012, https://doi.org/10.1139/p2012-044

[21] H. Youssef and N. A. Al-Ghamdi, "Vibration of Gold Nano Beam in Context of Two-Temperature Generalized Thermoelasticity without energy dissipation," in International Conference on Thermal Engineering, 2017.

[22] H. M. Youssef, "Thermal shock problem of a generalized thermoelastic solid sphere affected by mechanical damage and thermal diffusion," Journal of Engineering and Thermal Sciences, Vol. 1, No. 1, pp. 1-16, Jun. 2021, https://doi.org/10.21595/jets.2021.21934

[23] D. Tzou and M.-T. M. H. Transfer, "Lagging Behavior," in Macro - to Microscale Heat Transfer, Chichester, UK: John Wiley \& Sons Ltd, 2014, pp. 61-124, https://doi.org/10.1002/9781118818275.ch2

[24] D. Grover, "Damping in thin circular viscothermoelastic plate resonators," Canadian Journal of Physics, Vol. 93, No. 12, pp. 1597-1605, Dec. 2015, https://doi.org/10.1139/cjp-2014-0575

Photo unavailable due to local cultural reasons
Eman Al-Lehaibi received Ph.D. form Umm Al-Qura University in 2012 in Applied Mathematics. She published more than 36 papers in international journals and more than 8 papers in international conferences. Moreover, she is a member in European Mathematical Society and American Mathematical Society. Eman was a Co-PI in 5 scientific projects which have been funded through Umm Al-Qura University. Now, Eman is a Professor of Applied Mathematics in Alith College- Umm Al-Qura University. 